\title{
Finite Element Analysis of Static and Dynamic Characteristics of Elevator Desk Structure Based on ANSYS Workbench
}

\author{
Chen Jinlong $^{1, ~ a ~}$, Shen Zhenqian ${ }^{1, b, *}$ \\ ${ }^{1}$ School of Mechanical Engineering, Tianjin Polytechnic University, Tianjin 300387, China \\ ${ }^{2}$ School of Electronics and Information Engineering, Tianjin Polytechnic University, Tianjin \\ 300387, China \\ a1510370103@stu.tjpu.edu.cn, bshenzhenqian@tjpu.edu.cn, \\ *corresponding author
}

Keywords: Elevating computer desk, Statics analysis, Modal analysis, ANSYS Workbench

Abstract: Through the SolidWorks software for elevator computer desk $3 \mathrm{~d}$ entity modeling, and using the ANSYS Workbench software to model the stress analysis of meshing and the applied load for elevating computer desk overall deformation and stress distribution, thus carries on the strength, stiffness and modal analysis, the model for the first six order natural frequency and modal vibration mode, the analysis of dynamic performance of the model. The analysis results show that the strength and stiffness of the computer table meet the design requirements, and verify that no resonance occurs during the working process.

\section{Introduction}

With the development of science and technology, computers have become a necessary tool for daily office work and study. However, a series of health problems may be caused by sitting for a long time. Also appear on the market at present can hoist computer desk to solve the above problems, but in terms of structure design because of the lack of theoretical guidance, even completely rely on experience to carry on the design, appears unavoidably deformation of mechanical structure, intensity is low; For some electric motor driven lifting computer desk, vibration, noise and other problems will occur, seriously affecting the performance.

In order to ensure that the lifting desk structure meets the design requirements, its mechanical structure can also meet the design requirements. In this paper, SolidWorks 3d design software is used to conduct parametric modeling of the lifting computer desk, and it is imported into the finite element simulation software ANSYS Workbench for structural static analysis and modal analysis. Through the study of the meshing of assembly, the applied load and constraints, and get the stress and deformation nephogram, and former 6 order natural frequency and modal vibration mode, in order to decide whether lifting computer desk in design has the stability of the structure. Due to the limitation of space, only the static and dynamic characteristics of the lifting desk are analyzed, so as to provide an analytical method without further optimization design. 


\section{The main structure of the elevating computer desk}

The lifting computer table consists of a bottom plate, guide bracket, lifting plate, traction shaft, motor and top fixing box. The guide bracket is fixed vertically to the base plate seat. The lifting plate can slide up and down along the guide bracket in cooperation with the straight bearing and guide bracket set on it. The traction axis is connected by the screw nut and the lifting plate. Through the rotation of the traction axis, the movement of the lifting plate up and down is controlled to adjust the height of the computer.

\section{Build a 3D mode}

The lifting plate can move up and down along the guide rail under the action of the screw pair. The effective distance of the lifting plate movement is $386 \mathrm{~mm}$. According to the theory of mechanics $^{[1]}$ the situation of work and computer desk, under the same load condition, lifts up to the highest position, deformation of the system is the largest, so choose the position as a research position. The $3 \mathrm{~d}$ model is shown in figure 1.

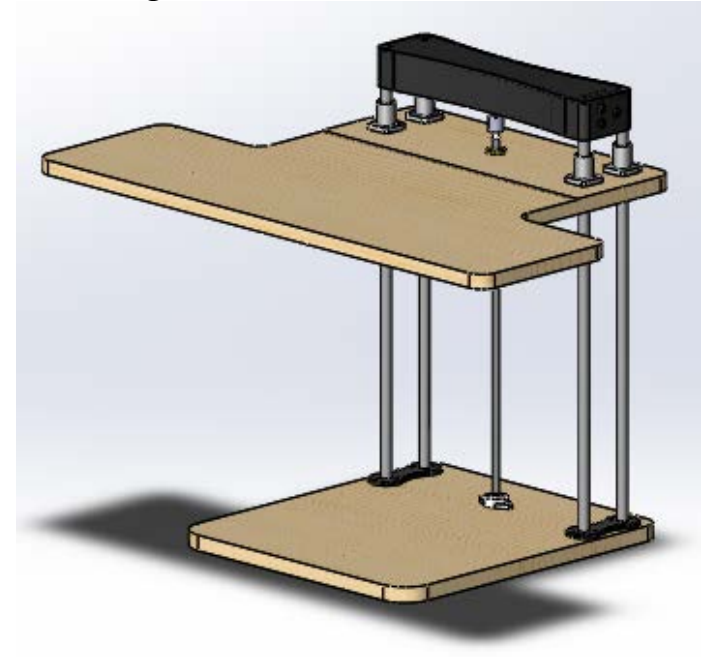

Figure 1: Elevator model of the computer desk.

\section{Establish the finite element model}

\subsection{Definition of material attributes}

The material of movable plate and bottom plate is pine. Its structural parameters are: density $398 \mathrm{~kg} / \mathrm{m}^{3}$, elastic modulus $12 \mathrm{Gpa}$, poisson ratio 0.3 . Nylon is selected for the top fixed box material, and its structural parameters are: density $1400 \mathrm{~kg} / \mathrm{m}^{3}$, elastic modulus $8.3 \mathrm{Gpa}$, poisson ratio 0.28 . The remaining materials are structural steel, whose structural parameters are: density $7850 \mathrm{~kg} / \mathrm{m}^{3}$, elastic modulus 200Gpa, poisson ratio 0.3.

\subsection{Finite element model and grid division}

Import the 3d overall Model of the elevating computer desk into Ansys Workbench in x_t file format, enter the Geometry environment first, reconstruct the Model, and then enter the Model environment to divide the grid. The default grid division is adopted, and Relevance Size is set to 50, Relevance Center is Medium, and Element is the default. Grid division generates 357014 nodes and 159761 units, as shown in figure 2. 


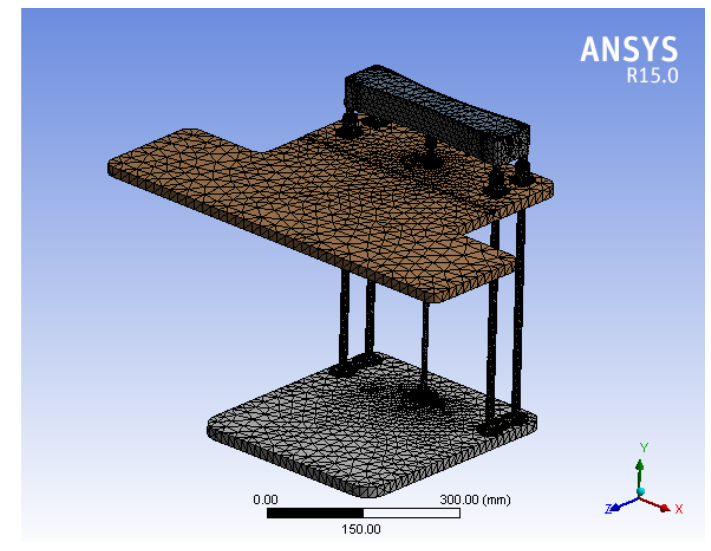

Figure 2: Grid division diagram.

\subsection{Constraint and load}

According to the actual working condition of elevating computer desk, the corresponding position in the process of lifting computer desk actual work restrictions, elevating computer desk in the practical work of base plate is flat on the table, so just need to plate fixed. Click supports, select Fixed Support, and then select the bottom of the bottom panel, which limits the position of the computer desk at work. Because the elevator computer desk under normal working condition lifting plate and guide bracket is fixed together, convenient to analysis and improve the efficiency of calculation, so set the contact type to Bonded (binding).

In the structural analysis, the mass of the moving plate and the attachment itself is treated as the self-weight load. Standard Earth Gravity is added along the direction of -y and 200N force is added on the lifting plate along the direction of $-\mathrm{y}$. See figure 3.

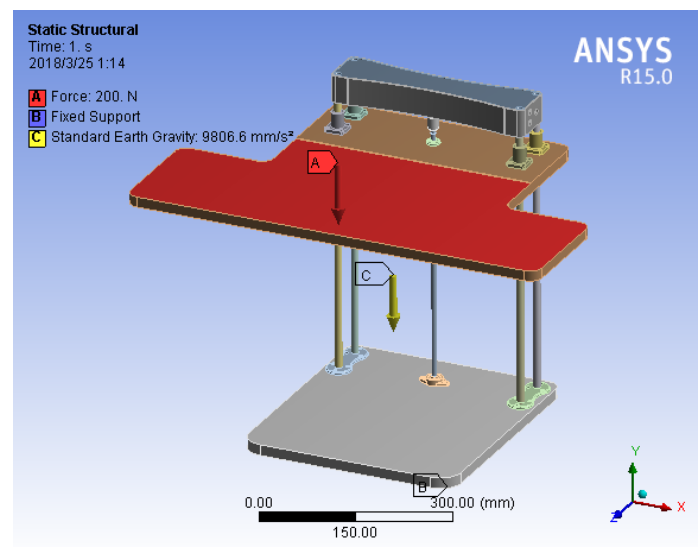

Figure 3: Imposes constraints and loads.

\section{Static analysis of lifting computer table}

\subsection{Fundamentals of structural statics analysis}

Structural statics are used to calculate the effects of structures under fixed and constant loads, regardless of the effects of inertia and damping.

The equation of static analysis is.

$$
[K]\{x\}=[F]
$$


Where, $[\mathrm{K}]$ is the stiffness matrix; $\{\mathrm{x}\}$ is the displacement vector; $[\mathrm{F}]$ is static load. Assuming that the material is linear elasticity and the structure is small deformation, then $[\mathrm{K}]$ is a constant matrix and must be continuous.

\subsection{Analysis on strength and rigidity of lifting computer desk}

After the constraint and load were applied, the Solution parameters were added to select Total Deformation cloud map during Deformation. Results the equivalent Stress of equiva-lent Stress and Total displacement of the Solution lifting computer table were analyzed. By the stress nephogram, the maximum stress is located in the linear bearing contact surface with the board, the maximum stress is $24.839 \mathrm{MPa}$, guide bracket and linear bearing contact position and the stress of the screw nut and screw position in about $20 \mathrm{MPa}$, other parts of the stress is small, can be neglected. See figure 4 . The ultimate tensile strength of structural steel is $460 \mathrm{MPa}$, and the structural strength fully meets the requirements of use.

The maximum displacement is located at the edge of the moving plate, and the maximum value is $0.97233 \mathrm{~mm}$, as shown in figure 5 . The deformation of the lifting computer desk is mainly concentrated on the moving board, that is, the position of bearing the working load. The deformation of other parts is minimal, and the static rigidity meets the requirements, which can meet the requirements for use.

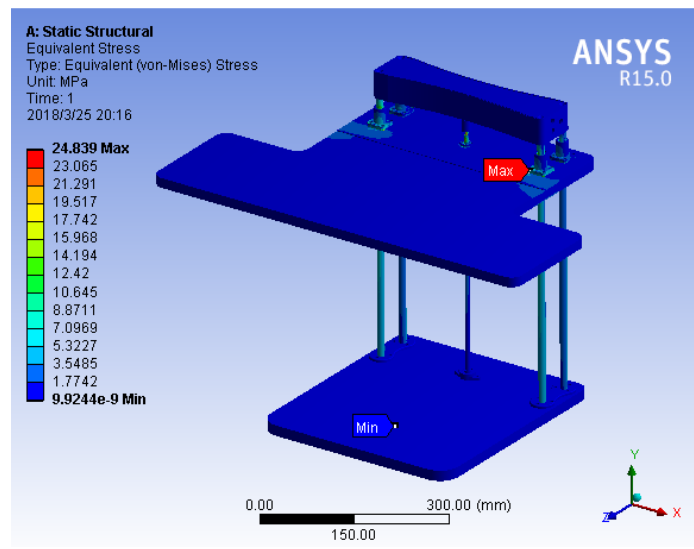

Figure 4: Stress nephogram.

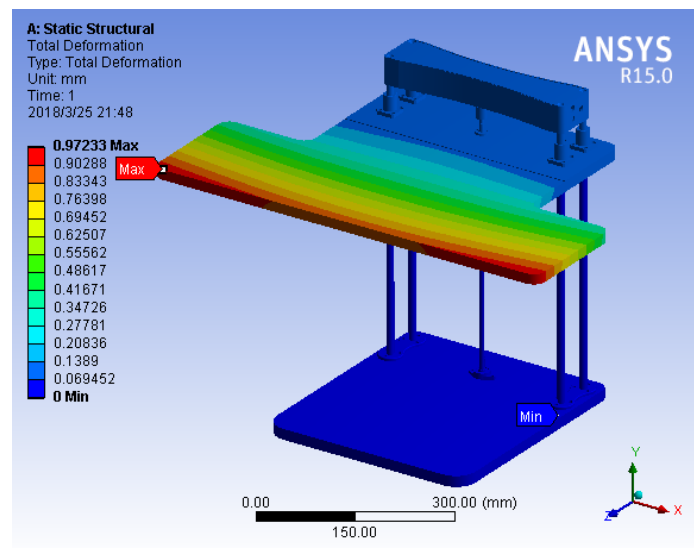

Figure 5: Deformation nephogram.

\section{Modal analysis of elevating computer table}

\subsection{Modal analysis theory}

Modal analysis is the linear time-invariant system vibration differential equations of physical coordinate transformation for the modal coordinates, the equations of decoupling, as a set of modal coordinates and the modal parameters which is used to describe the independent equation of, so that the modal parameters of the system.

The dynamics equation of motion of the problem

$$
[M]\{\ddot{x}\}+[K]\{x\}=\{0\}
$$

The free vibration of the structure is simple harmonic vibration, that is, the displacement is sine function

$$
\mathrm{x}=\mathrm{x} \sin \omega t
$$

Into the equations of motion 


$$
\left([K]-\omega^{2}[M]\right)\{x\}=\{0\}
$$

$[\mathrm{K}]$ is the stiffness matrix; $\{\mathrm{x}\}$ is the displacement vector; The equation of the characteristic value of $\omega_{\mathrm{i}}{ }^{2}$, is the circular frequency of vibration and natural frequency of $\mathrm{f}=\omega_{\mathrm{i}} / 2 \pi$, eigenvalue corresponding eigenvector $\{\mathrm{x}\}_{\mathrm{i}}$ for natural vibration frequency $\mathrm{f}=\omega_{\mathrm{i}} / 2 \pi$ corresponding vibration mode of $\pi / 2^{[2]}$.

\subsection{Modal analysis of elevating computer table}

Elevator computer desk complex structure, adopt the method of test analysis to its difficulty, and considering the actual working condition, adopt the method of experimental modal analysis is not economic, so using the finite element numerical simulation to conveniently and accurately on the modal analysis.

According to the actual working process of the lifting computer desk, it can be seen that under the normal working condition (the motor drives the moving plate up and down), it is mainly affected by the motor rotation. In order to understand elevator computer desk in motor incentive effect, on the basis of static analysis Modal analysis of the elevator computer desk, using Modal analysis module of ANSYS Workbench Modal for the previous six order natural frequency ${ }^{[3]}$, as shown in table 1. The corresponding modal modes are shown in figure 6 to figure 11.

Table 1 First 6 natural frequencies.

\begin{tabular}{|c|c|}
\hline Mode & Frequency(Hz) \\
\hline 1 & 27.889 \\
\hline 2 & 33.604 \\
\hline 3 & 40.62 \\
\hline 4 & 90.795 \\
\hline 5 & 132 \\
\hline 6 & 233.86 \\
\hline
\end{tabular}

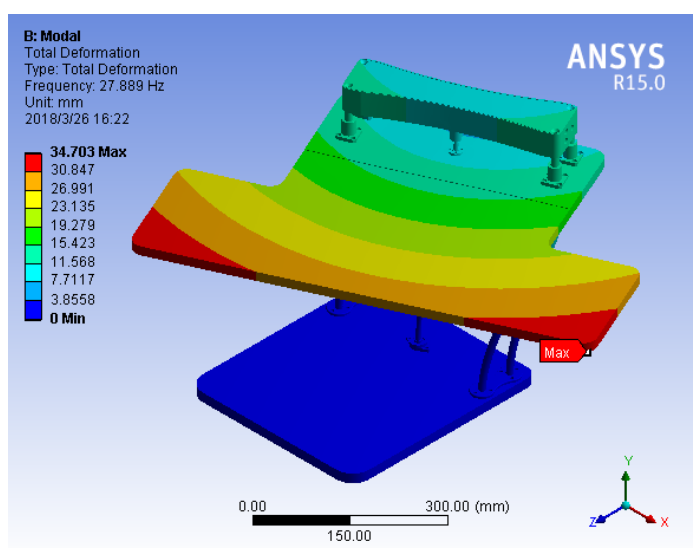

Figure 6: 1 order vibration mode

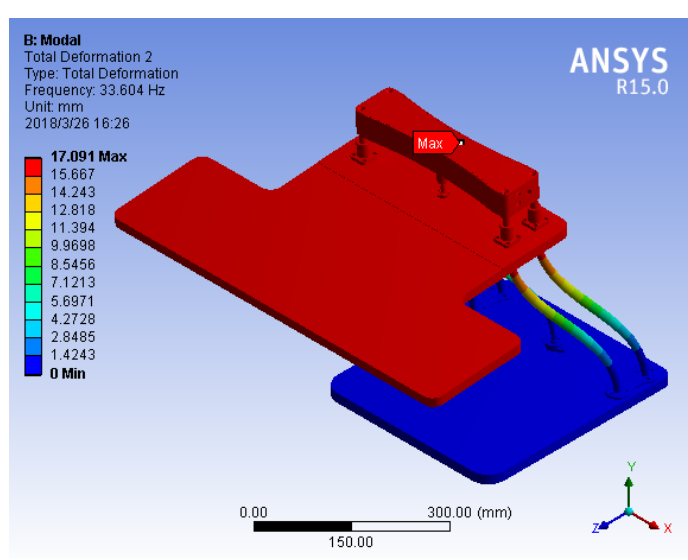

Figure 7: 2 order vibration mode 


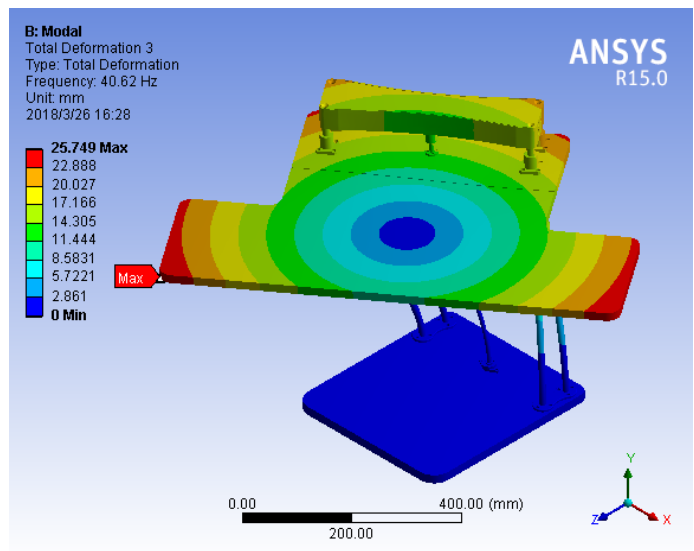

Figure 8: 3 order vibration mode

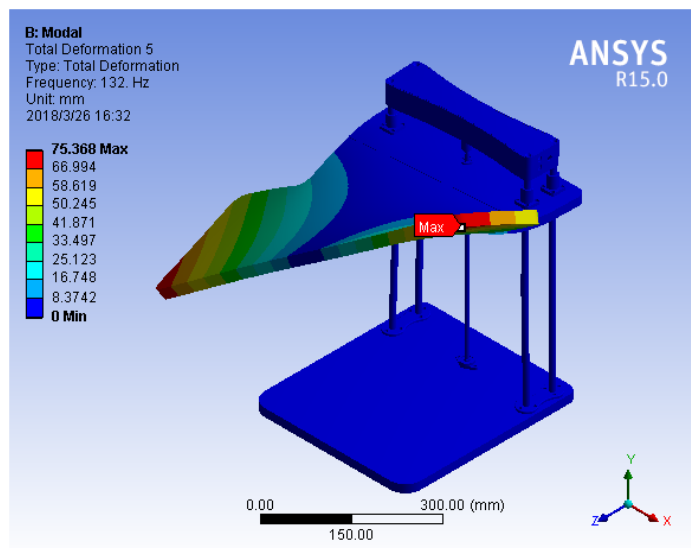

Figure 10: 5 order vibration mode

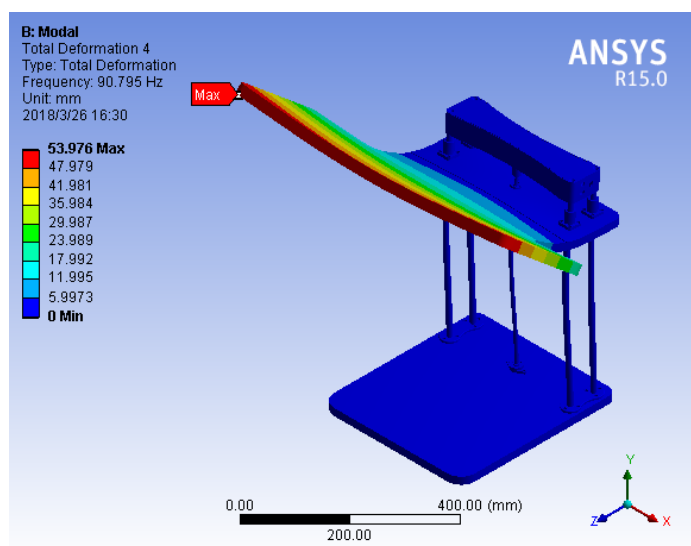

Figure 9: 4 order vibration mode

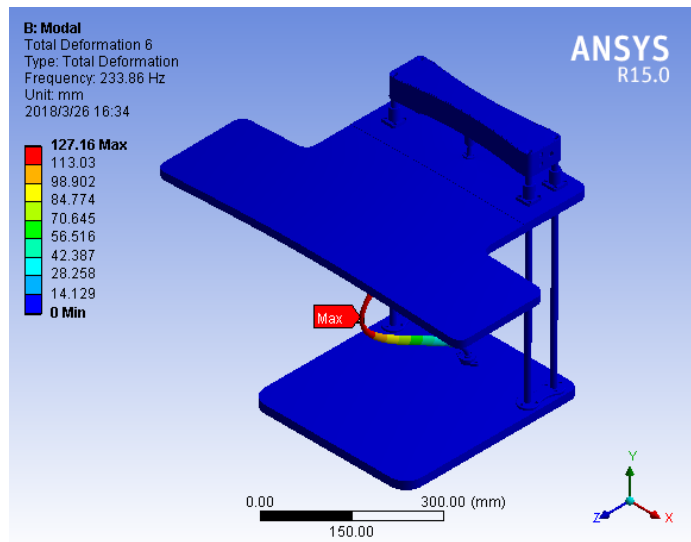

Figure 11: 6 order vibration mode

Can be seen from the figure shape modes and deformation, the first three order mainly guide bracket and reverse bending deformation of a ball screw, 4 order is active plate bending deformation, the fifth order is torsional deformation of the plate, 6 order is bending deformation of a ball screw. This provides a basis for the lifting computer table to avoid the natural frequency and avoid resonance in actual work ${ }^{[4]}$.

The computer table uses the permanent magnet synchronous motor ${ }^{[5-6]}$ as the power source, the rated speed is $n=110 \mathrm{r} / \mathrm{min}$, the motor operating frequency is $f=1.83 \mathrm{~Hz}$, and the natural frequency of the first order is $27.889 \mathrm{~Hz}$. The modal frequencies of each order gradually increase with theincrease of the mode order. Accordingly, the design of the structure of the computer desk effectively to avoid the resonance region, its inherent frequency far away from the work the resonant frequency, so the design of the structure of the computer desk is reasonable, not happen resonance damage ${ }^{[8]}$.

\section{Conclusions}

Through SolidWorks software, the 3d model of the lifting computer desk was established, and the model was imported into ANSYS Workbench to conduct structural strength, stiffness and modal analysis in Workbench. The following conclusions were drawn:

1) according to the results of the simulation platform of computer desk guide bracket, linear bearings and screw nut stress is larger, the deformation of the active plate is larger, the results accord with the actual, analysis method is feasible.

2) on the basis of statics analysis modal analysis was carried out on the elevator computer desk, it is concluded that the first six order natural frequency and vibration mode, lifting computer desk 
showed normal conditions there will be no danger of resonance.

3) the use of SolidWorks and ANSYS Workbench for collaborative simulation and performance analysis of the elevating computer desk can shorten the design cycle and save costs.

\section{Acknowledgements}

2017 national university student creative entrepreneurship training program (201710058028).

\section{References}

[1] Department of theoretical mechanics, Harbin Institute of Technology. Theoretical mechanics [M]. Beijing: education press, 2009:6-105.

[2] Liu Jiang.ANSYS 14.5 Workbench mechanical simulation example [M]. Beijing: machinery industry press, 2014:133-179.

[3] Wu Tianxing, Hua Hongxing. Mechanical vibration [M]. Beijing: tsinghua university press, 2014:100-101.

[4] Liu Zhengshi, Gao Ronghui, Chen enwei. Fundamentals of mechanical dynamics [M]. Beijing: advanced education press, 2011:44-50.

[5] Wang Chenggang, Yan Furong, He Fan, Sun Baokun, Qu Linshuai, you yingqiang. Vibration control analysis of permanent magnet synchronous motor [J]. Mechanical design and research. 2016,43 (11): 1-3.

[6] Ma Shumei, Ke Yulin, Li Aiping, Liu Xuemei. Finite element analysis of static and dynamic characteristics of main shaft of high efficiency permanent magnet synchronous motor based on ANSYS [J]. Mechanical design. 2013,30 (2) :82-85.

[7] Chen Jing, Yang Zolong, An Yongcheng, Wu Zhenyang. Analysis of dynamic characteristics and topological optimization of columns based on ANSYS [J]. Mechanical design. 2015,32 (6): 61-65.

[8] Zhang Gongxue, Meng Zhiqiang, Zhu Jjun. Fuzzy reliability theory analysis of resonance machinery [J]. Mechanical design.2001 (1): 27-29. 